over the study period. From 2000 to 2012, the annual hospitalization rate for gout increased from 12.2 to 16.7 per 100000 adults $(p=0.0038$ ). The increase was most pronounced in males aged 65 and above and over the last three years of the study. From 2009 to 2012 the inflation-adjusted health care costs for gout hospitalizations increased from 5.21 to $8.1510^{5}$ USD. The duration of hospitalizations also increased from 3 to 5 days median 2000 and 2012 respectively ( $p=0.021$ ). Only a minority of patients, 19 to $27 \%$, received ULT the 6 months preceding their hospitalization, without any obvious secular trend.

Conclusions: Incidence of hospitalization for primary gout is increasing substantially in Sweden over the last decade and this is reflected in the health care costs. The main part of this increase consists of males aged 65 and above. Only a fourth of the patients were on ULT preceding the hospitalization. These findings are further emphasized by the fact that the total amount of days for somatic inpatient care in WSHCR decreased by $9 \%$ from 2002 (1 267900 days, mean duration 5,7 days) to 2012 ( 1151630 days, mean duration 4,9 days). The findings in this study reflects increasing incidence of the gout disease and an ageing population but also a considerable lack of treatment.

Disclosure of Interest: None declared

DOI: 10.1136/annrheumdis-2017-eular.1825

\section{OP0263 TRANS-ANCESTRAL META-ANALYSIS IDENTIFIES 13 NEW LOCI ASSOCIATED WITH SERUM URATE LEVELS}

T.R. Merriman ${ }^{1}$, M. Cadzow ${ }^{1}$, R. Topless ${ }^{1}$, D. Mount ${ }^{2}, \mathrm{H}_{\text {. Choi }}{ }^{3}$, Y. Okada ${ }^{4}$, J. Boocock ${ }^{5}$, E. Stahl ${ }^{6}{ }^{1}$ University of Otago, Dunedin, New Zealand; ${ }^{2}$ Brigham and Women's Hospital; ${ }^{3}$ Harvard Medical School, Boston, United States; ${ }^{4}$ Osaka University, Osaka, Japan; ${ }^{5}$ University of California Los Angeles, Los Angeles; ${ }^{6} \mathrm{Mt}$ Sinai School of Medicine, New York, United States

Background: Serum urate is an important biomarker for gout disease and kidney function. Genome-wide association study (GWAS) meta-analyses have identified 28 loci in European and East Asian population samples. Combined analysis of these summary data across populations offers the opportunity to discover new serum urate associations through greater sample size and power, and trans-ancestral analyses provide the opportunity for fine-mapping associations with greater resolution given differing linkage disequilibrium patterns between populations.

Objectives: To conduct a meta-analysis of European and East Asian serum urate GWAS.

Methods: Summary statistics from European $(\mathrm{N}=110,238)$ (Kottgen et al. 2013) and east Asian $(\mathrm{N}=21,268)$ (Okada et al. 2012) meta-analyses were obtained. We used ImpG v1.0 to impute the results into 1000 Genomes phase 3 variants, and performed sample-size weighted $z$-score meta-analysis. Linkage-disequilibrium (LD)-independent variants with $\mathrm{P}_{\text {META }}<5 \times 10-8$, not in $\mathrm{LD}(\mathrm{r} 2<0.1)$ with previously identified regions, were considered novel serum urate loci. We used functional partitioned LD score regression on all associated loci in the European GWAS to identify SNP-heritability enriched tissue-specific regulatory regions, for use as functional priors in PAINTOR fine mapping analyses to identify putative causal variants.

Results: The trans-ancestral meta-analysis of European and East Asian GWAS revealed nine new serum urate-associated loci $\left(P_{\text {META }}<5 \times 10^{-8}\right)$. Three of the new loci are located in the 11q12.3-13.2 region near the established SLC22A11/12 locus. Additional novel loci are located near the FGF5, LNC00603, HLA-DQB1, B4GALT1, BICC1 and USP2 genes. Tissue-focused functional partitioning of SNP-heritability indicated the strongest enrichments of kidney, GI and liver tissues $\left(\mathrm{P}<10^{-7}\right)$, among other significant tissues. Trans-ancestral meta-analysis and functional fine-mapping decreases the numbers of SNPs in causal variant credible sets, and for example pinpoints the rs17632159 SNP as likely causal (posterior $\mathrm{P}>0.9$ ) at the TMEM171/174 locus.

Conclusions: Meta-analysis of existing GWAS increases power and leads to the identification of nine new loci associated with serum uric acid levels. Increased resolution in trans-ancestral GWAS, with functional annotation enrichments, improves fine-mapping of serum urate GWAS loci.

\section{References:}

[1] Köttgen A, et al. Genome-wide association analyses identify 18 new locis associated with serum urate concentrations. Nature Genet. 2013;45:145-54.

[2] Okada Y, et al. Meta-analysis identifies multiple loci associated with kidney function-related traits in east Asian populations. Nature Genet. 2012;44:904-9. Disclosure of Interest: None declared

DOI: 10.1136/annrheumdis-2017-eular.4611

\section{OP0264 CAN MODERATE ALCOHOL INTAKE LOWER THE RISK OF MYOCARDIAL INFARCTION AND MORTALITY EVEN AMONG GOUT PATIENTS?}

H.K. Choi ${ }^{1}$, N. Lu ${ }^{1,2}$, S.K. Rai ${ }^{1}$, S.F. Keller ${ }^{1}$, A.M. Jorge ${ }^{1}$, Y. Zhang ${ }^{1,2} .{ }^{1}$ Division of Rheumatology, Allergy, and Immunology, Massachusetts General Hospital; ${ }^{2}$ Clinical Epidemiology Research and Training Unit, Boston University School of Medicine, Boston, United States

Background: While alcohol is a well-established risk factor for gout, many prospective studies have consistently found that moderate alcoholic consumption is associated with a $25-40 \%$ reduced risk for coronary heart disease (CHD) and death. As such, the American Heart Association (AHA) suggests that "if you drink alcohol, do so in moderation". As gout is associated with an increased risk of $\mathrm{CHD}$ and premature mortality, the potential benefits of drinking in moderation may also be applicable to gout patients provided that their gout is under control with other measures.

Objectives: To examine the relation between alcohol intake and the risk of acute myocardial infarction (AMI) and all-cause mortality among incident gout patients in a general population context.

Methods: We conducted a cohort study using data from an electronic medical record database representative of the UK general population, collected between 1995 and 2015. The exposure of interest was the first alcohol intake measured after gout diagnosis, and our endpoints were incident cases of AMI as well as all-cause mortality. Stratifying by sex, we calculated the hazard ratios (HR) of these endpoints according to alcohol intake categories (i.e., 0, 1-9, 10-24, 25-42, and $>42$ UK alcohol units/week [1 unit=8gm]), adjusting for age, smoking status, body mass index, duration of gout, comorbidities, and medication use. We performed a spline model analysis using 4 knots of the alcohol intake categories, hypothesizing a J-shaped relation as observed in general population studies.

Results: Among 55,584 gout patients (78\% male, mean age of 63 years), 1,332 developed AMI and 8,362 died over a mean follow-up of 5.6 years. Compared with men who did not drink alcohol after gout onset, men who drank alcohol had a lower risk of AMI as well as mortality in a J-shaped manner (Figure 1, left panel). The multivariable HRs for developing AMI were 1.0, 0.76 (95\% confidence interval [Cl], 0.63-0.93), $0.68(0.55-0.83), 0.69(0.53-0.89)$, and $0.71(0.52-0.95)$ for alcohol use categories of $0,1-9,10-24,25-42$, and $>42$ units/week, respectively, and the corresponding HRs for mortality were $1.0,0.77(95 \% \mathrm{Cl}, 0.71-0.82), 0.71$ (0.66-0.77), $0.74(0.66-0.82)$, and $0.89(0.78-1.01)$ (Figure 1, left panel). While alcohol consumption levels and sample sizes were smaller among women, the $\mathrm{J}$-shaped relation was more obvious and showed a significantly increased risk of mortality in the top consumption category (HR=1.68; 95\% Cl, 1.09-2.61) (Figure 1 , right panel).
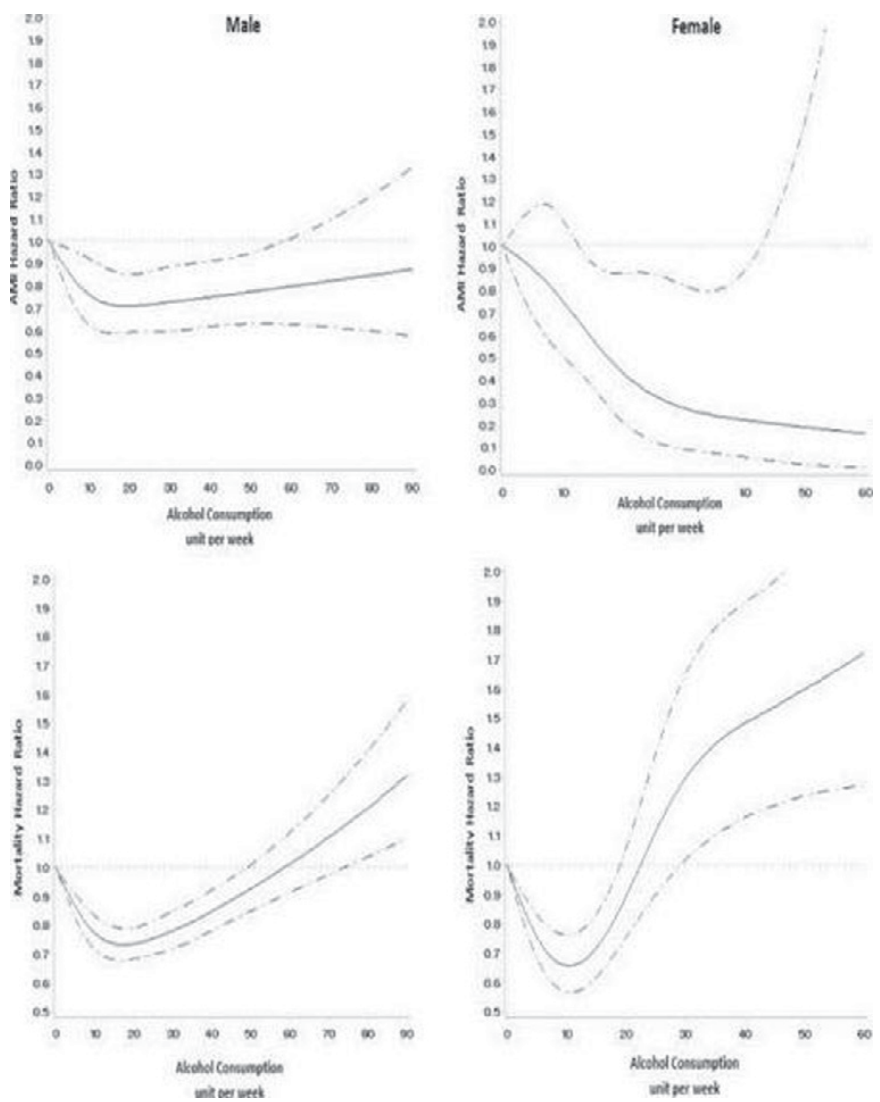

Conclusions: This general population-based study indicates that moderate alcohol intake is associated with a lower risk of AMI as well as all-cause mortality among gout patients, similar to many general population studies. These findings suggest that the AHA recommendation about moderate alcohol use may also be applicable to gout patients for their cardiac health and improved survival, provided that their gout is under control with other measures.

Acknowledgements: This project was supported in part by $\mathrm{NIH}$ grant P60-AR047785 and NIH grant R01-AR-065944.

Disclosure of Interest: None declared

DOI: 10.1136/annrheumdis-2017-eular.6906 